\title{
Ações do enfermeiro na atenção básica para prevenção do suicídio
}

\author{
Nayra Karoline Neco da Silva ${ }^{1}$ \\ Claudia Maria Sousa De Carvalho ${ }^{2}$ \\ Juliana Macêdo Magalhães² \\ José Arnaldo Moreira de Carvalho Junior ${ }^{2}$ \\ Bernadete Vieira da Silva Sousa ${ }^{3}$ \\ Wanderson Carneiro Moreira ${ }^{4}$
}

\begin{abstract}
A atenção básica representa o nível de atenção à saúde no qual há potencial para identificação e intervenção precoce no risco de suicídio. Os objetivos, neste estudo, foram descrever as ações realizadas pelo enfermeiro da atenção básica para prevenção do suicídio e discutir o processo de trabalho voltado para prevenção. É estudo do tipo exploratório-descritivo, de abordagem qualitativa, realizado com enfermeiros da Estratégia Saúde da Família. Utilizouse a técnica de análise de conteúdo para o tratamento dos dados. Por meio dos resultados, revelou-se que as ações para prevenção do suicídio na atenção básica necessitam ser inseridas no processo de trabalho de enfermeiros.
\end{abstract}

Descritores: Prevenção; Enfermeiras e Enfermeiros; Suicídio.

\footnotetext{
${ }^{1}$ Enfermeira, Hospital Prontomed, Teresina, PI, Brasil.

${ }^{2}$ MSc, Professor, Centro Universitário Uninovafapi, Teresina, PI, Brasil.

${ }^{3}$ Enfermeira, Vigilância Sanitária, Teresina, PI, Brasil.

${ }^{4}$ Aluno do curso de graduação em Enfermagem, Centro Universitário Uninovafapi, Teresina, PI, Brasil.
} 


\title{
Nursing actions in primary care to prevent suicide
}

Primary care is the health care level at which the risk of suicide can be identified, with the possibility of early intervention. The objectives in this study were to describe the primary health care nurses' potential to prevent suicide and discuss the work process focused on prevention. An exploratory and descriptive study with a qualitative approach was undertaken, involving nurses from the Family Health Strategy. The content analysis technique was used to treat the data. The results revealed that the actions to prevent suicide in primary health care need to be included in the nurses' work process.

Descriptors: Prevention; Nurses; Suicide.

\section{Aciones de la enfermera en la atención primaria para prevecion del suicídio}

\begin{abstract}
A atenção básica representa o nível de atenção à saúde no qual há potencial para identificação e intervenção precoce no risco de suicídio. Os objetivos, neste estudo, foram descrever as ações realizadas pelo enfermeiro da atenção básica para prevenção do suicídio e discutir o processo de trabalho voltado para prevenção. É estudo do tipo exploratório-descritivo, de abordagem qualitativa, realizado com enfermeiros da Estratégia Saúde da Família. Utilizouse a técnica de análise de conteúdo para o tratamento dos dados. Por meio dos resultados, revelou-se que as ações para prevenção do suicídio na atenção básica necessitam ser inseridas no processo de trabalho de enfermeiros.
\end{abstract}

Descriptores: Prevención; Enfermeros; Suicidio.

\section{Introdução}

A prática do suicídio ocorre desde a Antiguidade, porém, sua relevância, em estudos, pouco tem sido explorada, visto que envolve tabus, preconceitos e incógnitas em torno de sua história.

O autoextermínio é considerado fenômeno multidimensional, de repercussões inespecíficas, e representa, atualmente, importante problema de saúde pública, visto que afeta não somente de maneira individual como, também, coletiva. Outro fator relevante a ser levado em consideração diz respeito ao crescimento em nível global, considerando o aumento das taxas de suicídio em $60 \%$ nos últimos 50 anos $^{(1)}$.

Relativamente ao comportamento suicida, estimase que as tentativas superem o número de suicídios consumados em pelo menos 10 vezes. Tentativas de suicídio devem ser encaradas com seriedade, como sinal de alerta, revelando a influência de fenômenos psicossociais inespecíficos e complexos. Desse modo, pela existência de tentativa, é possível prever a ocorrência de suicídio(1-2).

Embora a questão relativa ao suicídio seja algo preocupante e que, em face a diversas indagações relacionadas ao problema, se evidencie abundante produção científica acerca da temática, as pesquisas já realizadas parecem não contribuir consideravelmente para a redução da incidência dos casos, levando-se em conta o crescimento desordenado no número de tentativas e suicídios findos em todo o mundo ${ }^{(3)}$.

Ainda que, no suicídio, estejam envolvidas as mais variadas questões como, por exemplo, as genéticas socioculturais e psicodinâmicas, entre os grupos mais vulneráveis às tentativas de suicídio estão pessoas que possuem algum transtorno mental, como quadro de depressão, esquizofrenia ou transtorno bipolar(1). Nesse sentido, embora a maior parte das tentativas de suicídio e suicídios consumados seja praticada por aqueles que possuem algum diagnóstico de transtorno mental, é primordial destacar que não 
devem ser negligenciados os casos nos quais não há relação com nenhum transtorno mental.

Entre os anos 2002 e 2012, o total de ocorrências de suicídio, no Brasil, passou de 7.726 para 10.321, o que aponta crescimento de $33,6 \%$ dos casos, dado alarmante, considerando o fato de que esse aumento foi superior ao crescimento da população do país no mesmo período, no total de $11,1 \%$. Das taxas de mortes violentas, foi a de maior crescimento decenal, superando largamente os homicídios, com 2,1\%, e a mortalidade ocorrida em acidentes de transporte, com 24,5\%(4).

Nesse prisma, do ponto de vista da prevenção do suicídio, as equipes de saúde que atuam no âmbito da atenção básica, por representarem o primeiro nível dos recursos de atenção à saúde, possuem elevado potencial para o desenvolvimento de estratégias e ações que propiciem a identificação e intervenção precoce em casos de risco de suicídio. Tal potencialidade das equipes da atenção básica tem, entre suas justificativas, o modelo de organização definido pelo Ministério da Saúde (MS), no qual a atenção básica constitui a porta de entrada preferencial do usuário ao sistema de saúde, promovendo acolhimento, vínculo e responsabilização pela atenção às necessidades das pessoas que buscam esse nível da assistência(5).

Contudo, no que concerne às ações para prevenção do suicídio, tais práticas não têm sido rotineiramente evidenciadas no desenvolvimento do processo de trabalho desses profissionais. Verifica-se que algumas ações voltadas à prevenção do suicídio somente são contempladas em equipes, nas quais há profissionais que se identificam com a área, revelando lacunas na disposição do modelo de organização dessas ações no âmbito da atenção básica.

Entende-se que a prevenção do comportamento suicida não é tarefa fácil. Para que se possibilite a redução desse risco, a detecção precoce de pessoas com ideação suicida e a intervenção apropriada são fatores fundamentais ${ }^{(6)}$.

É necessário que os serviços de saúde estejam cada vez mais bem estruturados e capazes de promover resolutividade no que se refere a agravos, tais como tentativas de suicídio. Desse modo, tornase imperioso que o enfermeiro da atenção básica seja capaz de identificar precocemente os sinais e sintomas inatos do paciente, dedicando a atenção merecida ao mesmo, tendo em vista que, por meio do acolhimento adequado, é possível promover a garantia da qualidade do atendimento direcionado e eficácia do tratamento.

\section{Método}

Pesquisa de caráter exploratório-descritiva, de natureza qualitativa, realizada em Unidades Básicas de Saúde (UBS) e Unidades de Saúde da Família (USF), com implantação da Estratégia Saúde da Família (ESF), no município de Teresina, PI, Brasil. Para a pesquisa, foram selecionadas as seguintes UBSs e USFs: UBS Oséas Sampaio (Matadouro), USF Mafrense, USF Nova Brasília e ESF Poty Velho. A escolha das referidas unidades de saúde deu-se pela facilidade de reconhecimento do território onde estão mapeados os dispositivos terapêuticos que compõem a rede assistencial de saúde.

Os critérios de inclusão definidos para os participantes foram: ser enfermeiro, atuante na atenção básica há, no mínimo, um ano e aceitar participar da pesquisa por meio da assinatura do Termo de Consentimento Livre e Esclarecido (TCLE). Não houve restrições quanto ao sexo dos participantes.

Assim, participaram da pesquisa seis enfermeiras da ESF que atenderam os critérios de inclusão. Vale destacar que, entre os profissionais enfermeiros que atenderam aos critérios de inclusão, três recusaramse a participar da pesquisa e três encontravamse de férias de suas atividades. Contudo, mesmo considerando o grau de dificuldade de adesão de enfermeiros à pesquisa, entende-se que, com o número de participantes envolvidos no estudo, foi possível responder ao objeto de estudo desta pesquisa e alcançar os objetivos estabelecidos, pois a saturação das falas foi evidenciada claramente nas entrevistas realizadas.

A coleta de dados aconteceu no mês de abril de 2015 e foi realizada por meio de entrevistas semiestruturadas, com o seguinte roteiro: código do participante, data da realização da entrevista, nome do entrevistador e tempo de duração da entrevista. Para melhor caracterização dos participantes, foram definidos critérios como idade, sexo, tempo de formação, tempo de atuação na ESF e especialização em Saúde Mental (SM). Ao final do roteiro, havia a questão norteadora: quais as ações realizadas por enfermeiros na atenção básica para prevenção do suicídio?

As entrevistas foram realizadas individualmente, em espaço reservado, na própria unidade básica, a fim de garantir segurança e privacidade ao entrevistado. O registro das respostas deu-se por meio de equipamento eletrônico tipo gravador, após o esclarecimento da pesquisa e consentimento do participante. Contudo, as entrevistas somente tiveram início após Parecer de aprovação do Comitê de Ética e 
Pesquisa (CEP), sob o CAAE: 41748015.1.0000.5210. Além disso, foram respeitados todos os aspectos éticos e legais da Resolução $n^{\circ} 466 / 12$, que trata de pesquisas envolvendo seres humanos.

A análise dos dados foi realizada por meio da técnica de análise de conteúdo, à luz do referencial teórico de Minayo. Tal ferramenta consiste em recurso técnico para análise de dados provenientes de mensagens escritas ou transcritas. Assim, na análise de conteúdo da pesquisa, propôs-se descobrir os núcleos de sentido que puderam compor comunicação cuja presença ou frequência significaram alguma coisa para o objetivo analítico ${ }^{(7)}$. Dados esses discutidos em categoria temática.

\section{Resultados e Discussão}

A partir da aplicação de instrumento de entrevista semiestruturada para a coleta dos dados, com questões fechadas sobre os participantes da pesquisa, foi possível traçar o perfil desses profissionais, conforme descrito na Figura 1.

\begin{tabular}{|c|c|c|c|c|}
\hline Participantes & Sexo & Idade & $\begin{array}{c}\text { Tempo de } \\
\text { profissão }\end{array}$ & $\begin{array}{c}\text { Especialização } \\
\text { em SM }\end{array}$ \\
\hline P1 & Fem. & 45 & 15 anos & Não \\
\hline P2 & Fem. & 39 & 10 anos & Não \\
\hline P3 & Fem. & 26 & 4 anos & Não \\
\hline P4 & Fem. & 50 & 30 anos & Não \\
\hline P5 & Fem. & 36 & 12 anos & Sim \\
\hline P6 & Fem. & 45 & 16 anos & Não \\
\hline
\end{tabular}

Figura 1 - Características das enfermeiras participantes do estudo. Teresina, PI, Brasil, 2015

É importante mencionar que, durante o período da coleta de dados, sabendo das medidas relacionadas ao processo de trabalho do enfermeiro, previstas na política nacional de prevenção do suicídio, buscou-se, na análise dos dados, descrever as ações realizadas por essas profissionais no sentido da prevenção, identificando, por meio de seus depoimentos, se as mesmas realizam ou não medidas preventivas em relação ao suicídio. Dessa forma, a análise dos depoimentos convergiu para a formação de uma categoria temática - o enfermeiro da atenção básica e as ações para prevenção do suicídio: desafios do processo de trabalho.

É interessante destacar que, na análise e discussão dessa categoria, buscou-se estabelecer comparação entre a realidade estudada na literatura e o modelo de trabalho de enfermeiros da ESF, no sentido da prevenção do suicídio.
O enfermeiro da atenção básica e as ações para prevenção do suicídio: desafios do processo de trabalho

Apesar da relevância do presente estudo para a saúde pública, a composição daquilo que pode ser analisado durante a pesquisa apresenta-se como fator limitante e deve ser levada em consideração.

Tendo em vista que as equipes da ESF desenvolvem seu processo de trabalho no território onde vivem os usuários, bem como planejam suas ações a partir da identificação de riscos e necessidades da população, as ações para prevenção do suicídio devem fazer parte da agenda de trabalho das equipes, constituindo atividades inerentes ao cotidiano das mesmas. O enfermeiro, como integrante da equipe de saúde da família, tem, entre suas atribuições, a responsabilidade de desenvolver o acolhimento aos usuários por meio da escuta de suas necessidades, possibilitando a identificação de riscos e vulnerabilidades, permitindo o planejamento do cuidado(5).

Contudo, embora a equipe da ESF e, particularmente, o enfermeiro como membro da equipe, tenha atribuições ampliadas de cuidados às necessidades da população adscrita, em relação às ações para prevenção do suicídio, ainda não se verificam ações organizadas no processo de trabalho desse profissional que possibilitem a identificação de riscos e vulnerabilidades relacionadas ao suicídio, conforme exposto pelas participantes, a seguir.

Atualmente, eu ainda não desenvolvo na minha unidade nenhuma atividade de prevenção do suicídio. Eu não sei assim, pelo fato do suicídio ser uma questão que a gente ainda não aborda muito (P3)

[...] na nossa equipe, pelo menos, nós não temos nenhuma ação [...] (P1).

Em contextos nos quais a prevenção do suicídio deve compor parte da gama de atividades inerentes ao cotidiano das equipes de saúde da ESF, tornase extremamente importante que o enfermeiro, integrante fundamental na assistência ao usuário, planeje ações efetivas e permanentes a respeito da prevenção do suicídio.

De acordo com as falas acima, evidenciou-se que não existe planejamento específico voltado à temática proposta, bem como ações efetivamente preventivas implementadas pelos profissionais que desenvolvem seu trabalho no âmbito da atenção básica. A ausência dessas ações foi notória nos relatos das participantes da pesquisa, visto que, de acordo com as mesmas, não há ações concretas nas UBSs em que elas desenvolvem seu processo de trabalho. 
Nos serviços de saúde, a atuação das equipes na verificação de fatores de risco para o suicídio, bem como a utilização de estratégias preventivas para essa condição, pode promover maior eficácia no que diz respeito à prevenção do suicídio. Em estudo realizado anteriormente, evidenciou-se que mais de $75 \%$ das vítimas de suicídio procuraram um serviço de atenção primária à saúde no ano de sua morte e, entre esse grupo de pessoas, $45 \%$ delas procuraram as UBSs no mesmo mês em que cometeram suicídio(3).

O primeiro contato com o paciente é extremamente importante. A abordagem calma, aberta, de aceitação e de não julgamento, por parte do enfermeiro, é fundamental para facilitar a comunicação. Criar esse vínculo é, por si só, importante passo para reduzir o nível de desespero do paciente suicida ${ }^{(8)}$.

Na atenção básica, em nível da Estratégia Saúde da Família, a gente tem pouca abordagem sobre o assunto junto aos usuários. O que a gente tem, no momento, é quase nada... A gente não tem, tipo assim, um direcionamento pra resolver esses problemas, a prevenção em si não é realizada $(\mathrm{P} 5)$.

Eu ainda não tenho tanta abertura para falar sobre suicídio. Para mim, ainda é um tema difícil de conversar com o paciente, pelo fato da gente não ser preparado para lidar com essa situação-limite, então eu ainda tenho, talvez, uma dificuldade (P3).

Eu não me sinto preparada para lidar com isso. Vejo o suicídio como um desespero, é angustiante, evito até falar no assunto. Me sinto muito angustiada, sem saber o que fazer. A saída que vejo é encaminhar para outro profissional (P6).

É de suma importância que o enfermeiro esteja disponível e atento para possíveis sinais e sintomas de pacientes com ideação suicida, sendo esse profissional capaz de realizar escuta qualificada, com foco apenas no paciente e nas questões que o colocaram em situação de completo desespero e total desesperança. Esse profissional deve ser capaz de deixar de lado possíveis julgamentos e vieses que surgem em face de situações-limite como o suicídio.

Notabiliza-se, no estudo, que, além da falta de preparo para lidar com essa situação tão delicada e complexa, existem também incógnitas relacionadas ao tema para o próprio profissional que, por não saber lidar com a situação, finda por vivenciar o sentimento de angústia, que pode ser reflexo da dificuldade de enxergar possíveis soluções para o problema.

O fato do profissional de saúde da atenção primária estar disponível e acessível favorece o estabelecimento do elo vital entre a comunidade e o sistema de saúde. Tais fatores podem culminar em importante passo para a prevenção do suicídio(9) ${ }^{(9)}$ detecção precoce e o tratamento apropriado dessas condições são de extrema relevância no que tange à prevenção.

Nesse contexto, por meio das relações humanas, o enfermeiro observa o paciente, experenciando as mais variadas emoções que ocorrem entre eles, tais como dor, mal-estar, tristeza, alívio ou esperança(10).

[...] a gente percebe quando a gente conversa com o paciente, a gente percebe que ele tem alguma depressão, ou ele apresenta sinais, né, como choro, ansiedade, insônia [...] (P2).

Atualmente, eu costumo apresentar aos agentes de saúde, que existe um serviço especializado no Lineu Araújo. A partir desse conhecimento, que eu obtive através de um treinamento no Caps Norte, nós passamos a orientar os agentes de saúde. Já tive a oportunidade de entregar folhetos para as familias, que a gente sabe que em Teresina tem um índice muito alto de suicídio (P4).

O enfermeiro da atenção básica muitas vezes possui forte relação com o paciente em virtude do estabelecimento do vínculo, que é fortalecido a cada nova consulta de enfermagem. Tal ferramenta permite a ele identificar mudanças negativas no comportamento de quem recebe o atendimento, aspecto esse evidenciado no relato de uma das participantes acima. O que pode promover a percepção do diagnóstico de enfermagem que caracterize o risco de suicídio.

Já as ações caracterizam-se como medida de intervenção potencialmente eficaz, podendo culminar na preservação de uma vida, ainda sejam que realizadas de maneira não tão específica, como relatado na fala de $\mathrm{P} 4$, que visa o encaminhamento do paciente a local com profissionais especializados em com questões relacionadas ao suicídio.

No Brasil, a proposta de atenção integral realizada na atenção básica, bem como o atendimento de pessoas em linhas de cuidado, constituem cenário favorável para a organização e execução das políticas de atenção ao suicídio e suas diretrizes, tendo em vista a integralidade da assistência. Relativamente a programas de prevenção comunitários que tenham como intuito acolher os usuários desse sistema, tais como o programa Hiperdia, o qual contribui para prevenção e tratamento de hipertensão arterial e diabetes, resultados satisfatórios são evidenciados ${ }^{(11)}$.

Entretanto, no que se refere especificamente ao suicídio, concorda-se que, ainda que existam ações de prevenção a esse tipo de violência autoinfligida em âmbito nacional, não são encontradas referências específicas de programas para prevenção do 
suicídio, embora possam estar em desenvolvimento ações locais, ainda não suficientemente divulgadas

Nas falas das participantes a seguir foi possível identificar a falta de preparo para lidar diretamente com questões ligadas ao suicídio. Tal comprometimento de habilidade pode ser reflexo da ausência de conhecimento específico ligado à ciência da SM, tendo em vista que, das seis participantes da pesquisa, apenas uma relatou possuir o título de especialista nessa área. As falas ainda revelam possíveis motivos pelos quais ações no sentido da prevenção do suicídio não têm sido postas em prática, sendo unanimidade a ausência de treinamentos voltados para essa problemática.

Nunca recebemos nenhum treinamento para lidar com esse tipo de problema. Tem os outros treinamentos básicos do dia a dia: tuberculose, hanseníase, a questão da gestante, pré-natal, isso é muito explorado. Mas com relação focalizada apenas na questão do suicídio, nunca teve nada voltado para gente, não (P1).

A gente é muito treinado para lidar com tuberculose, hanseníase, mais nessas condições de saúde pública mesmo. Não que o suicídio não seja uma preocupação de saúde pública, é uma preocupação, mas eu acho que a gente ainda não é preparado para lidar com o suicídio. Acho que falta realmente isso, treinamento (P3).

Com relação à prevenção do suicídio, nós não temos nenhum programa que tenha sido lançado pela fundação para trabalhar com a prevenção do suicídio, nem houve nenhum treinamento. O que acontece é junto aos outros programas que a gente faz os atendimentos, que é os grupos de diabéticos, hipertensos, pré-natal e Bolsa Família (P2).

As ações educativas indicadas acerca da abrangência da prevenção universal do suicídio devem fazer parte do processo de trabalho do enfermeiro, no âmbito da atenção básica, considerando que ele já realiza atividades relacionadas à educação em saúde envolvendo patologias amplamente discutidas e esplanadas, como as relatadas pelas três participantes acima.

Nessa perspectiva, é importante que haja parceria entre gestores, profissionais de saúde e enfermeiros, de forma que cada um compreenda exatamente qual o seu papel. É necessário que os gestores de saúde tenham real noção da dimensão do problema e, consequentemente, da importância da disponibilização de meios de informação, treinamento e capacitação desses profissionais de saúde, para que eles estejam preparados para lidar adequadamente com o problema e sejam capazes de não somente identificar possíveis sinais e sintomas inatos de pacientes com ideação suicida, mas, também, aptos para realizar as devidas intervenções nas crises.

É primordial enfatizar que o enfermeiro da atenção básica compreenda a relevância da busca de informações pertinentes relacionadas a esse tema. Desse modo, é imprescindível que o profissional busque conhecimento acerca desse assunto. O enfermeiro deve inserir no seu processo de trabalho planejamento sistemático, seguido de ações educativas efetivas no sentido de promover a prevenção do suicídio, aliando o conhecimento específico necessário à prática de medidas eficazes que visem ações de prevenção.

\section{Conclusão}

Em relação às ações para prevenção do suicídio, objeto de estudo nesta pesquisa, verificou-se que, embora enfermeiros percebam a necessidade de planejamento de intervenções quanto a essa problemática, considerando a gravidade de tal fato, ainda não se verificam ações organizadas e executadas homogeneamente entre as agendas de trabalho dos profissionais enfermeiros participantes deste estudo. Tal deficiência é apontada pelos profissionais entrevistados por diversas causas, que incluem desde a falta de identificação profissional com a área e inabilidade para enfrentar essa questão à falta de recursos, por meio de treinamento e capacitação sobre o suicídio, que possibilitem ao enfermeiro desenvolver habilidade e competência técnica para lidar e intervir nesse problema. Por meio dos resultados deste estudo, sinaliza-se, ainda, a necessidade de ampliar e fortalecer a rede de cuidados para questões relacionadas ao suicídio, de modo que os profissionais estejam preparados para desenvolver ações de prevenção e possam intervir, também, em situações de crise em face de suicídio. Para tanto, é necessário o devido envolvimento e compromisso de gestores, profissionais de saúde e comunidade.

\section{Referências}

1. Botega NJ. Suicídio: Saindo da sombra em direção a um plano nacional de prevenção. [editorial] Rev Bras Psiquiatr. 2007;29(1):7-8.

2. Luz L. Suicídio de médicos: a necessidade de uma reflexão. [Dissertação de Mestrado em Bioética]. São Paulo: Centro Universitário São Camilo; 2010. 52 p.

3. Abreu KP, Lima MAD, Kohlrausch E, Soares JF. Comportamento suicida: fatores de risco e intervenções preventivas. Rev Eletr Enferm. [Internet]. 2010 [Acesso 
12 nov 2014];12(1):195-200. Disponível em: http://www. fen.ufg.br/fen_revista/v12/n1/pdf/v12n1a24.pdf

4. Walselfisz JJ. Mapa da Violência 2014: os Jovens do Brasil. Rio de Janeiro: Flacso; 2014.

5. Ministério da Saúde (BR) Secretaria de Atenção à Saúde Departamento de Atenção Básica. Política Nacional de Atenção Básica. Brasília: Ministério da Saúde; 2012.

6. Ministério da Saúde (BR). Prevenção do suicídio: manual dirigido a profissionais das equipes de saúde mental [Internet]. Brasília: Ministério da Saúde; 2006. [Acesso 2 set 2014] Disponível em: http://bvsms.saude. gov.br/bvs/publicacoes/manual_editoracao.pdf

7. Minayo MCS. O desafio do conhecimento: pesquisa qualitativa em saúde. 28. ed. São Paulo/Rio de Janeiro: Hucitec-Abrasco; 2007. 202 p.

8. Ministério da Saúde (BR). Portaria №1.876 de 14 de agosto de 2006. [Internet]. [Acesso 19 de nov 2014] Disponível em: http://bvsms.saude.gov.br/bvs/ saudelegis/gm/2006/prt1876_14_08_2006.html

9. Organização Mundial da Saúde (OMS). Prevenção do Suicídio: um manual para profissionais de saúde em atenção primária [Internet]. Genebra: OMS; 2000 [Acesso 12 nov 2014]. Disponível em: http://www.who.int/mental_ health/prevention/suicide/en/suicideprev_phc_port.pdf.

10. Navarro C, Martinez M. Attitudes of nursing professionals towards suicidal behavior: influence of emotional intelligence. Rev. Latino-Am. Enfermagem [Internet]. nov-dez 2012 [Acesso 20 nov 2014]; Disponível em: http://www.scielo.br/pdf/rlae/v20n6/pt_19.pdf

11. Conte M, Meneghel NS, Trindade AG, Ceccon RF, Hesler LZ, Cruz CW, et al. Programa de Prevenção ao Suicídio: estudo de caso em um município do sul do Brasil. Ciênc Saúde Coletiva. 2012;17(8):2017-26. 